\title{
Loading and Maintenance Doses of Digoxin in Patients with Normal Renal Function and Those with Severely Impaired Renal Function
}

JOHN G. WAGNeR, Ph.D. Ann Arbor, Mich.

$\mathrm{T}_{\mathrm{s}}^{\mathrm{n}}$ HE clinician may find the equations ing and difficult to follow. However, pharmacokinetic theory is of little use unless those familiar with the theory show how application of the theory is of practical importance. This paper is an attempt to do so with a drug that has presented many problems with respect to dosing and toxicity. It is really not important that the clinician completely understand how the results were calculated; it is important for him or her to remember the results for each drug for which the equations were applied.

The arguments are presented in the text, and the equations in the Appendix, so that those familiar with pharmacokinetic theory can apply them to other drugs and produce still more practical information for the busy clinician. The principal role of the clinician, with respect to the aspect of medicine discussed in this report, is to determine the relationship between clinical effect and dose of a drug. The principal roles of the pharmaco-

From the College of Pharmacy and the Upjohn Center for Clinical Pharmacology, The University of Michigan, Ann Arbor, Mich. 48104. Supported in part by U.S. Public Health Service Grant 5 P11 GM 15559 and in part this work was performed pursuant to Contract No. FDA 69-22 with the Public Health Service, Food and Drug Administration, Department of Health, Education and Welfare.

July, 1974 kineticist, with respect to the same aspect, are: (1) to develop models from observed measurements such as plasma concentrations; (2) to show the relationship between the clinical effect and some observed or model-calculated values; and (3) to make recommendations concerning the appropriate way to obtain maximum benefit from a given dose of the drug and, at the same time, reduce the chance of toxicity to as low a probability as possible.

Jelliffe $^{1}$ has suggested that it is not necessary to change the loading dose of digoxin in patients with renal failure. Chung ${ }^{2}$ recommends that the loading dose of digoxin be decreased to from one third to two thirds of the usual loading dose. Reuning et al. ${ }^{3}$ suggested "that the loading dose of digoxin for such patients should be decreased to one half to two thirds of the normal loading dose in order to achieve blood levels in the desired therapeutic range." This author agrees with the figure of two thirds if blood levels are the appropriate criterion. However, this author believes that the report of Reuning et al. ${ }^{3}$ indicates that the average amount of drug in the second compartment of the two-compartment open model is the appropriate criterion; using this criterion, the loading dose should be the same in severely impaired renal patients as it is in normal patients.

Reuning et al. ${ }^{8}$ also stated, "The optimal 
loading dose for digoxin does not depend on how rapidly the drug is excreted but rather upon how large a body space must be 'filled up' to a therapeutic level by the initial dose." This statement is erroneous, since for any model, any drug, or any criterion the loading dose must always be related to the rate of elimination of the drug as well as the volume which needs to be filled. In the two-compartment open model, the volume that needs to be "filled up" is what is called the volume of distribution steady state, $\nabla_{d . .}$ As seen below, $\nabla_{d \text {., }}$ for severely impaired renal patients averages 364 liters and for patients with normal renal function, it averages 520 liters. Hence, on the basis of volume effects only, one would calculate that the loading and maintenance doses in severely impaired renal patients should both be decreased to 70 per cent $(364 / 520 \times 100)$ of the doses for patients with normal renal function.

But this is an incorrect estimate, since the rate of elimination of digoxin in the two types of patients must also be taken into consideration in estimating loading and maintenance doses. If you have a leaky bucket and you try to fill the bucket up to a certain level with water, it is obvious that the amount of water needed is dependent upon the size of the holes in the bucket! In pharmacokinetics, the most important factor determining the size of the loading dose is the fraction of each dose that is eliminated from the body in each dose interval. This fraction is equal to $\beta \tau$, or its equivalent, $1.443 t_{\xi} / \tau$, where $t_{t}$ is the half-life of elimination and $\tau$ is the uniform dosing interval.

The recommended loading doses of digoxin for patients with normal renal function in the literature are too high for the following reasons. First, the recommendations are based upon the one compartment open model, whereas Reuning et al. $^{3}$ and others have shown that plasma concentration data following intravenous administration of digoxin are explained by the two-compartment open model. The loading dose based on the two-compartment open model will always be less than the loading dose based on the one-compartment open model. Secondly, the elimination half-life of digoxin used in the calculation of the loading has frequently been too high. Third, the loading dose needed is directly proportional to the maintenance dose of digoxin required to produce the desired clinical effect. It has been reported ${ }^{4}$ that 75 per cent of patients are maintained on $0.25 \mathrm{mg} /$ day or $0.375 \mathrm{mg}$ average (0.5 $\mathrm{mg}$ one day and $0.25 \mathrm{mg}$ the next day), 20 per cent of patients are maintained on 0.5 $\mathrm{mg} /$ day, and 5 per cent on $0.75 \mathrm{mg} /$ day. Hence for a new patient with normal renal function, it appears that one should calculate the loading dose on the basis of a maintenance dose of $0.25 \mathrm{mg} / \mathrm{day}$, follow this loading dose with maintenance doses of $0.25 \mathrm{mg} /$ day, and then adjust the maintenance dose on an individual patient basis to obtain the desired clinical effect in that patient.

The two-compartment open model is schematically shown in scheme I below.

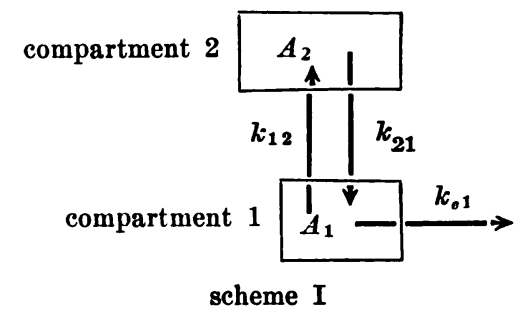

where $k_{12}, k_{21}$, and $k_{e 1}$ are first-order rate constants, $A$ represents the amount of drug at time $t$, and the subscripts 1 and 2 refer to the compartment number. The amount of drug, $A_{1}$, divided by the apparent volume of compartment $1, V_{1}$, is the "plasma concentration" of the drug at time $t$.

Reuning et $a .^{3}$ showed that the left ventricular ejection time index and the

The Journal of Clinical Pharmacology 
$\mathrm{Q}-\mathrm{S}_{2}$ interval correlated with the fraction of the digoxin dose in compartment 2 (and not with the fraction of the dose of digoxin in compartment 1 ). Thus, the response to digoxin is correlated with "tissue levels" and not with plasma levels, as might be expected, since cardiac tissue would be expected to be included in compartment 2 and not in compartment 1.

The maintenance doses of digoxin in patients with severe renal failure must be reduced since their $k_{e 1}$, hereafter called $\left(k_{e 1}\right)_{r}$, is smaller (about one half) than the $k_{e 1}$, hereafter called $\left(k_{e 1}\right)_{n}$, of patients with normal renal function. Since the response to digoxin is proportional to the average amount of drug in compartment $2, \bar{A}_{2}$, at steady state, then one should calculate the loading dose on the basis that severely impaired renal patients will have the same value of $\overrightarrow{A_{2}}$ at steady state as patients with normal renal function. That is, one should calculate to make $\left(\bar{A}_{2}\right)_{r}=$ $\left(\bar{A}_{2}\right)_{n}$, where the subscript $r$ refers to severely impaired renal patients and the subscript $n$ refers to patients with normal renal function. This type of calculation gives a totally different result than a calculation based on making the steady-state plasma concentrations equal [i.e., $(\bar{C})_{r}=$ $\left.(\bar{C})_{\mathrm{n}}\right]$. Hence, the problem with the recommendation of Reuning et al. ${ }^{3}$ was use of the plasma level criterion rather than the $\vec{A}_{2}$ criterion. This is unfortunate, since it was they who showed that $A_{2}$ was the important factor in the response.

\section{Methods}

We can set up three criteria and determine what the result would be by each criterion. These criteria are as follows:

Criterion 1. The maintenance dose ratio $R_{1}$, equal to $D_{r} / D_{n}$, will be such as to provide equal steady-state plasma concentrations in both severely impaired renal patients and in patients with normal renal function [i.e., $(\bar{C})_{r}=(\bar{C})_{n}$ ].

July, 1974
Criterion 2. The maintenance dose ratio $R_{2}$ will be such as to provide equal steadystate average amounts of drug in the body, $\bar{A}_{b}$ (where $\bar{A}_{b}=\vec{A}_{1}+\bar{A}_{2}$ ) [i.e., $\left.\left(\bar{A}_{b}\right)_{r}=\left(\overline{A_{b}}\right)_{n}\right]$.

Criterion 3. The maintenance dose ratio $R_{3}$ will be such as to provide equal steadystate average amounts of drug in compartment 2, $\bar{A}_{2}$ [i.e., $\left(\bar{A}_{2}\right)_{r}=\left(\bar{A}_{2}\right)_{n}$ ].

Pharmacokinetic equations given in the Appendix show how the ratios $R_{1}, R_{2}$, and $R_{3}$ are calculated.

Reuning et al. $^{3}$ reported parameter values of digoxin for the model shown in scheme I, both for patients with severely impaired renal function and patients with normal renal function. From their values, weighted mean parameter values were calculated. Also, from these weighted mean parameter values, various other needed parameters were calculated.

\section{Results}

The weighted mean parameter values and other needed parameters, calculated from the data presented by Reuning et al., ${ }^{3}$ are shown in Table I. Definitions of the calculated parameters are given in the footnotes to Table I.

The ratios of maintenance doses, $R_{1}, R_{2}$, and $R_{3}$, needed to meet criteria 1 through 3 were calculated using the equations in the Appendix and are tabulated in Table II. One can see from Table II that, to produce equal steady-state plasma concentrations, the maintenance dose of digoxin in patients with severely impaired renal failure should only be one third of the maintenance dose of digoxin given to patients with normal renal function. However, to produce both equal steady-state average amounts of digoxin in the body and equal steady-state average amounts of digoxin in compartment 2 , the maintenance dose of digoxin in patients with severely impaired renal function should be only about one half (round-off of $\mathbf{0 . 4 6}$ and 0.47 ) of the maintenance dose of 
TABLE I

Weighted Mean Parameter Values for Digoxin and Parameters Derived Therefrom

\begin{tabular}{|c|c|c|}
\hline \multirow[b]{2}{*}{ Parameter* } & \multicolumn{2}{|c|}{ Parameter values } \\
\hline & $\begin{array}{l}\text { Patients with severely } \\
\text { impaired } \\
\text { renal function }\end{array}$ & $\begin{array}{l}\text { Normals } \\
\text { subjects }\end{array}$ \\
\hline & \multicolumn{2}{|c|}{ Weighted Means } \\
\hline$k_{12}(\mathrm{hr}-1)$ & 0.45 & 0.56 \\
\hline$k_{21}(\mathrm{hr}-1)$ & 0.113 & 0.15 \\
\hline$k_{e 1}(\mathrm{hr}-1)$ & 0.040 & 0.081 \\
\hline$\nabla_{1}$ (liters) & 73. & 110. \\
\hline \multirow{2}{*}{$\nabla_{d \text { extrap }}$ (liters) } & 360. & 840. \\
\hline & \multicolumn{2}{|c|}{ Derived Values } \\
\hline$\alpha(h r-1)$ & 0.595 & 0.775 \\
\hline$\beta(h r-1)$ & 0.0076 & 0.0156 \\
\hline$t_{\xi}(\mathrm{hr})$ & 91.2 & 44.2 \\
\hline$\nabla_{\text {d., (liters) }}$ & 364. & 520. \\
\hline$\nabla_{d_{\text {area }}}$ (liters) & 384. & 571. \\
\hline Correction factor (C.F.) & 0.947 & 0.914 \\
\hline Accumulation ratio & 5.2 & 2.4 \\
\hline \multicolumn{3}{|c|}{ 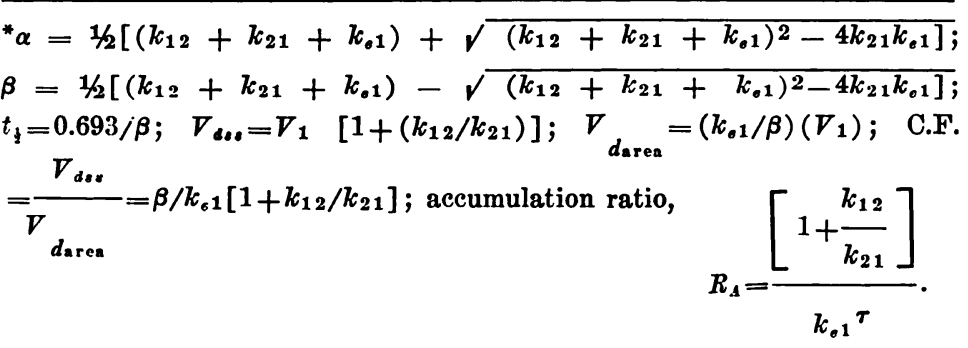 } \\
\hline
\end{tabular}

digoxin given to patients with normal renal function. Because of the correlations reported by Reuning et al., ${ }^{3}$ the author believes that the proper ratio is given by criteria 2 and 3 and not by criterion 1.

As indicated by eq. (9) of the Appendix, the appropriate loading dose $D^{*}$ is equal to the drug accumulation ratio $R_{A}$ multiplied by the maintenance dose. Such a loading dose $D^{*}$ would with one dose provide the same average amount of drug in the body $\left(A_{b}\right)$ as would be ultimately attained if one gave no loading dose but only maintenance doses of size $D$ at once-a-day intervals. The calculated value of $R_{A}$ for patients with severely impaired renal function is 5.0 (round-off of 5.2 ), and the calculated value of $R_{A}$ for patients with normal renal function is 2.5 (round off of 2.4) (see Table I). Hence, for digoxin one has the result that the ratio of $\left(R_{A}\right)_{r} /\left(R_{A}\right)_{n}=5 / 2.5=2$, and the ratio of maintenance doses is $D_{r} / D_{n}=$ $0.5 / 1=1 / 2$; thus, the loading doses should 
TABLE II

Ratio of Maintenance Dose of Dogixin for Patients with Severely Impaired Renal Function to Maintenance Dose of Digoxin for Patients with Normal Renal Function

\begin{tabular}{ccccc}
\hline Criterion no. & \multicolumn{1}{c}{ Criterion } & $\begin{array}{c}\text { Ratio } \\
\text { code }\end{array}$ & $\begin{array}{c}\text { Maintenance } \\
\text { dose ratio }\end{array}$ & $\begin{array}{c}\text { Calculated } \\
\text { by appendix } \\
\text { equation }\end{array}$ \\
\hline 1 & $\begin{array}{c}\text { Equal steady-state plasma con- } \\
\text { centrations } \\
\text { Equal steady-state amounts of } \\
\text { drug in body } \\
\text { Equal steady-state amounts of } \\
\text { drug in compartment } 2\end{array}$ & $R_{1}$ & 0.33 & eq. (11) \\
3 & $R_{2}$ & 0.47 & eq. (12) \\
\hline
\end{tabular}

be the same in both severely impaired renal patients and patients with normal renal function. This result obviously results from the conclusion made in this report that the criterion 3 is the correct one and that criterion 2 gives essentially the same result for digoxin as eriterion 2.

If one argues that the steady-state plasma concentrations should be equal in the two types of patients, then the ratio of loading doses will be:

$$
\frac{\left(D^{*}\right)_{r}}{\left(D^{*}\right)_{n}}=\frac{\left(R_{A}\right)_{r} \cdot D_{r}}{\left(R_{A}\right)_{n} \cdot D_{n}} \simeq \frac{5}{2.5} \cdot(0.33)=0.67 .
$$

Thus, criterion 1 gives the result that the loading dose in patients with severely impaired renal function should be only two thirds of the loading dose given to patients with normal renal function. However, as stated formerly, this author does not believe that criterion 1 is the correct one for digoxin based on the data of Reuning et al. ${ }^{3}$

Table III gives calculated loading and maintenance doses of digoxin in patients with severely impaired renal function and calculated loading doses of digoxin in patients with normal renal function, based on the maintenance dose of digoxin required to produce the desired clinical effect in patients with normal renal function. Since obviously the clinician does not know a priori what maintenance dose of a corresponding normal patient to use, it appears safest to use the maintenance dose of $0.25 \mathrm{mg}$ in the normal patient; and this is why this dose and the other corresponding doses are underlined in Table III. This seems to be the "safest," since 75 per cent of patients are maintained on either 0.25 or $0.375 \mathrm{mg}$ digoxin per day. ${ }^{4}$ As stated formerly, the maintenance dose can then be adjusted later on an individual patient basis to produce the desired clinical effect of the drug. To be still "safer," the loading dose should be administered in several parts, as indicated in the footnote to Table III. Such a recommendation has been made formerly. ${ }^{5}$

\section{Discussion}

One must remember that when digoxin is administered orally, not all of the dose administered reaches the bloodstream of the patient. For the Lanoxin brand of digoxin tablets, an average of about 60 per cent of the administered dose reaches the bloodstream. ${ }^{6}$ For many generic tablets that have been studied, the percentage is lower and variable from brand to brand. ${ }^{7}$ Since most elinicians use the Lanoxin brand of digoxin tablets, the recommendations in Table III are only based on this brand. If the loading dose is 
TABLE III

Loading and Maintenance Doses of Digoxin Based on the Pharmacokinetic Analysis Presented in this Report*

\begin{tabular}{ccc}
\hline $\begin{array}{c}\text { Maintenance dose } \\
\text { in patients with } \\
\text { normal renal } \\
\text { function } \\
\text { (mg/day) }\end{array}$ & $\begin{array}{c}\text { Maintenance dose } \\
\text { in patients with } \\
\text { severely impaired } \\
\text { renal function } \\
\text { (mg/day) }\end{array}$ & $\begin{array}{c}\text { Recommended } \\
\text { loading dose for } \\
\text { both types of } \\
\text { patientst } \\
\text { (mg) }\end{array}$ \\
\hline 0.125 & 0.0625 & 0.315 \\
0.25 & $\underline{0.125}$ & $\underline{0.625}$ \\
\hline 0.375 & 0.1875 & 0.9875 \\
0.385 & 0.1925 & 0.9625 \\
0.50 & 0.25 & 1.25 \\
0.75 & 0.375 & 1.875 \\
\hline
\end{tabular}

* If the loading dose is administered intravenously the values in column 3 should be reduced by multiplying them by 0.6 .

† This loading dose should be divided into five equal parts and three parts given during the first day and two parts given during the second day; then the maintenance dose should be given on day 3 and all subsequent days until a dosage adjustment is made.

administered intravenously, all the figures in column 3 of Table III should be reduced by multiplying them by 0.6 .

The recommendations of this report agree with those of Jelliffe, ${ }^{1}$ but disagree with those of $\mathrm{Chun}^{2}$ and Reuning et al. ${ }^{3}$ Also, the loading doses recommended in column 3 of Table III are appreciably lower than those recommended in prior literature. For example, Ogilvie and Ruedy ${ }^{5}$ recommended a loading dose of $0.0075 \mathrm{mg}$ of digoxin per pound of lean body weight and a maintenance dose of 33.3 per cent of the loading dose if the blood urea nitrogen is $>20 \mathrm{mg} / 100 \mathrm{ml}$. For a 70-kg man $(154 \mathrm{lb})$, these recommendations are equivalent to a loading dose of $1.155 \mathrm{mg}$ and a maintenance dose of $0.385 \mathrm{mg} /$ day. This author believes that such an average man should initially receive a loading dose of $0.625 \mathrm{mg}$ of digoxin, as Lanoxin tablets orally, and maintenance doses of $0.25 \mathrm{mg} / \mathrm{day}$, and that the dose be then adjusted to produce the desired clinical effect in the particular patient.
The recommended loading dose of $0.9625 \mathrm{mg}$, corresponding to a maintenance dose of $0.385 \mathrm{mg} /$ day, is lower than the recommended loading dose of Ogilvie and Ruedy ${ }^{5}$ of $1.155 \mathrm{mg}$ since Ogilvie and Ruedy's loading dose is based on the onecompartment open model (i.e., their $\boldsymbol{R}_{A}$ value is $1 / \beta \tau=1 / 0.333=3$ ), while the value in Table III is based on the twocompartment model, so that $\boldsymbol{R}_{\boldsymbol{A}}$ is given by $\left[1+\left(k_{12} / k_{21}\right)\right] / k_{e 1} \tau=4.73 / 1.94 \simeq 2.5$. Smith, ${ }^{8}$ in a recent review, listed the usual oral maintenance doses of digoxin as $0.25-0.5 \mathrm{mg} /$ day and the average oral digitalizing dose as $1.25-1.5 \mathrm{mg}$. The ratios of loading dose/maintenance dose calculated from these values of Smith, namely, 3 to 5 , are again higher appreciably than the value of 2.5 used to prepare Table III and which is based on the pharmacokinetic analysis.

The effect of too high a loading dose relative to the maintenance dose is well illustrated by the data of Marcus et al. ${ }^{9}$ in the dog. Assuming that 30 per cent of the digoxin is excreted daily in the dog,

The Journal of Clinical Pharmacology 


\section{LOADING AND MAINTENANCE DOSES OF DIGOXIN}

they calculated that at the end of six days the amount of digoxin in the dog's body would be $0.412 \mathrm{mg}$ when the dog was dosed with $0.2 \mathrm{mg}$ digoxin/day. Instead of administering $0.412 \mathrm{mg}$ digoxin (which in this case is the appropriate loading dose), they administered a loading dose of $0.75 \mathrm{mg}$ digoxin followed by maintenance doses of $0.2 \mathrm{mg} /$ day. On the latter regimen, they calculated that at the end of six days the amount of digoxin in the body would be $0.476 \mathrm{mg}$ digoxin. They measured minimum blood concentrations each day just before dosing. The curve for the loading dose group peaked on day 2 at about $2.2 \mathrm{ng} / \mathrm{ml}$, then slowly decreased in staircase fashion to a concentration somewhat above $1 \mathrm{ng} / \mathrm{ml}$ at the end of the six days. The dogs that only received $0.2 \mathrm{mg} /$ day showed an ascending plasma level curve which at the end of the sixth day was still slightly less than $1 \mathrm{ng} / \mathrm{ml}$.

This author believes that such "overshoot" caused by too high a loading dose may cause many of the problems associated with digoxin therapy. Most reports have given only minimum plasma or blood levels measured just before doses. These will show the "overshoot" as indicated above. However, measurement of plasma levels during the absorption-distribution phase of digoxin would show that too high a loading dose would cause very high peak concentrations of digoxin at about 1 to 2 hours after oral dosing and even higher concentrations at time zero when the drug is administered intravenously. The author believes that these high peaks produced with too high loading doses may cause much of the toxicity observed; but, of course, this is only an opinion and would require experimental verification.

The equations in the Appendix are applicable to any drug. Since the parameter values are different for each drug, one must substitute the appropriate parameter values into the equations to calculate the ratios for a particular drug. Hence, the values reported here for digoxin should not be interpreted as being applicable to any other drug.

\section{Appendix}

Wagner et al ${ }^{10}$ gave the general eq. (1) for the average plasma (blood or serum) concentration at steady state, $\vec{C}$ :

$$
\bar{C}=\frac{F D}{\nabla K_{\tau}}
$$

where $F$ is the fraction of each maintenance dose $D$ which is absorbed, $\tau$ is the uniform dosing interval, $V$ is an apparent volume of distribution, and $K$ is a first-order rate constant for elimination from the central compartment. For each type of pharmacokinetic model, one must determine what $V$ and $K$ to substitute into eq. (1). Gibaldi et al. ${ }^{11}$ showed that for the two-compartment open model, $V$ is replaced by $V_{d_{\text {area }}}$ and $K$ is replaced by $\beta$, hence one obtains

$$
\bar{C}=\frac{F D}{\nabla_{d_{\mathrm{ares}} \beta \tau} .}
$$

If $\vec{A}_{b}$ is the average amount of drugs in the body at steady state (also equal to the amount of drug that goes into and out of the body during one dosage interval at the steady state), then for the one-compartment open model we may write

$$
\bar{A}_{0}=\frac{F D}{\beta \tau}
$$

since, for this simplest model, $K=\beta=k_{e 1}$ of the two-compartment model. Now in both the one- and two-compartment models, $\beta$ means the first-order rate constant estimated from terminal plasma concentrations such that the half-life of elimination, $t_{\mathrm{t}}$, is given by eq. (4) (the pharmacokinetic definition of $\beta$ is given as a footnote to Table I) :

$$
t_{1}=0.693 / \beta \text {. }
$$

Perrier and Gibaldi ${ }^{12}$ showed that for the two-compartment open model, $\bar{A}_{b}$ is given by

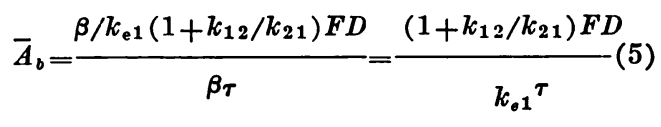

The drug accumulation ratio $R_{A}$, as given 


\section{WAGNER}

by Wagner, ${ }^{13}$ is the ratio of the average amount of drug in the body at steady state to the amount of drug absorbed following administration of one maintenance dose. This relationship is given in eq. (6) :

$$
R_{A}=\bar{A}_{b} / F D \text {. }
$$

Hence, for the one-compartment open model, by using eqs. (3) and (6), one obtains

$$
R_{A}=\frac{1}{\beta_{\tau}} \text {. }
$$

For the two-compartment open model, by using eqs. (5) and (6), one obtains

$$
R_{A}=\frac{\left[1+\frac{k_{12}}{k_{21}}\right]}{k_{e 1}^{\top}} .
$$

By comparing eqs. (5), (7), and (8), one can see that the quantity $\beta / k_{e 1}\left[1+\left(k_{12} / k_{21}\right)\right]$ is a "correction factor" which must be applied if the drug really obeys the two-compartment open model rather than the one-compartment open model.

If only maintenance doses of size $D$ are given at uniform time intervals $\tau$, one eventually reaches a steady state such that the average amount of drug $\bar{A}_{b}$ is in the body. The purpose of a loading dose is to quickly establish the amount of $\overline{A_{b}}$ in the body so that one does not have to wait a long time (i.e., administration of many small doses) before that level is reached. This is particularly important with long half-life drugs, since the time to reach, say, 95 per cent of the steadystate value $\bar{A}_{b}$ is directly proportional to the half-life of the drug. Hence, the appropriate loading dose $D^{*}$ is given by

$$
D^{*}=R_{A} \cdot D \text {. }
$$

That is, the proper loading dose $D^{*}$ is just the acoumulation ratio $R_{a}$ times the maintenance dose $D$.

It is also easily shown that the average amount of drug in compartment 2 of the twocompartment open model at steady state is given by

$$
A_{2}=\frac{k_{12} F D}{k_{21} k_{\mathrm{e} 1}{ }^{\top}} .
$$

\section{Criteria Discussed in Text}

Criterion 1. To make the steady-state plasma concentration in severely impaired renal patients, $(\vec{C})_{r}$, equal to the steady-state plasma concentration in patients with normal renal function, $(\bar{C})_{n}$, we must solve eq. (2) for $D$, then make a ratio of two of the resulting equations (the numerator for severely impaired and the denominator for normals). The result is

$$
R_{1}=\frac{D_{r}}{D_{n}}=\frac{\left(V_{d_{\text {area }}}\right)_{r} \cdot \beta_{r}}{\left(\nabla_{d_{\text {area }}}\right)_{n} \cdot \beta_{\mathrm{a}}}
$$

when $F_{r}=F_{n}$ and $\tau_{r}=\tau_{n}$. The subscript $r$ refers to the parameter value for patients with severely impaired renal function, and the subscript $n$ refers to the parameter value for patients with normal renal function.

Criterion 2. To make the steady-state average amount of drug in the body in severely impaired renal patients, $\left(\vec{A}_{b}\right)_{r}$, equal to the steady-state average amount of drug in the body of patients with normal renal function, $\left(\vec{A}_{b}\right)_{n}$, we must solve eq. (5) for $D$ and make a ratio of two of the equations as before. The result is

$$
R_{2}=\frac{D_{r}}{D_{n}}=\frac{\left[1+\frac{\left(k_{12}\right)_{n}}{\left(k_{21}\right)_{n}}\right]}{\left[1+\frac{\left(k_{12}\right)_{r}}{\left(k_{21}\right)_{r}}\right]} \cdot \frac{\left(k_{\theta 1}\right)_{r}}{\left(k_{, 1}\right)_{n}}
$$

when $F_{r}=F_{n}$ and $\tau_{r}=\tau_{n}$.

Criterion 3. To make the average amount of drug in compartment 2 at steady state in patients with severely impaired renal function, $\left(\bar{A}_{2}\right)_{r}$, equal to the average amount of drug in compartment 2 at steady state in patients with normal renal function, $\left(\bar{A}_{2}\right)_{n}$, we must solve eq. (10) for $D$ and make a ratio of two of the resulting equations as before. The result is

$$
R_{3}=\frac{D_{r}}{D_{n}}=\frac{\left(k_{12}\right)_{n}}{\left(k_{12}\right)_{r}} \cdot \frac{\left(k_{21}\right)_{r}}{\left(k_{21}\right)_{n}} \cdot \frac{\left(k_{31}\right)_{r}}{\left(k_{01}\right)_{n}}
$$

when $F_{r}=F_{n}$ and $\tau_{r}=\tau_{n}$.

It should be noted that the concept of drug accumulation as the buildup of the amount of drug in the body, as given by Wagner ${ }^{13}$ and in this report, is different than that of Krüger-Thiemer ${ }^{14,15}$ who considered drug accumulation as the buildup of drug concentrations. If one defines drug accumulation on the basis of buildup of drug concentration, then there will always be drug accumulation on multiple dosing of a drug unless the doses are spaced an infinitely long time apart. However, if one bases the definition on the amount

The Journal of Clinical Pharmacology 


\section{LOADING AND MAINTENANCE DOSES OF DIGOXIN}

of drug in the body related to the amount absorbed from each maintenance dose, then there is no accumulation providing the dosing interval is equal to, or greater than, the time constant of elimination of the drug (i.e., $\tau>1 / \beta)$. This makes much more sense to the author. The problem of side effects and toxicity arises when $\tau$ is made much less than $1 / \beta$, particularly in the use of long half-life drugs. There is a relationship, however, between loading dose recommendations made by Krüger-Thiemer ${ }^{14}$ and those indicated by eqs. (7) and (9) of this report. For the onecompartment open model with first-order absorption rate constant $k_{a}$ and elimination rate constant $K$, Krüger-Thiemer ${ }^{14}$ gave eq. (14) to calculate the loading dose:

$$
D^{*}=\frac{D}{\left(1-\mathrm{e}-k_{a} \tau\right)(1-\mathrm{e}-K \tau)} .
$$

When $k_{a}$ is large relative to $K$, eq. (14) reduces to

$$
D^{*}=\frac{D}{1-e-K \tau} .
$$

When $K_{\tau}>0.10$ (which occurs when the dosage interval $\tau$ is made equal to, or less than, the half-life of elimination, $0.693 / K$ ), then

$$
\frac{D^{*}}{D} \simeq \frac{1}{K_{\tau}}
$$

since, under such conditions, $\mathrm{e}^{-K \tau} \simeq 1-K_{\tau}$, and $1-\mathrm{e}^{-K \tau} \simeq K_{\tau}$. The result shown in eq. (16) is the same as obtained by substituting from eq. (7) into eq. (9).

Another advantage of defining drug accumulation in terms of average amounts rather than concentrations at a given time is that the average amount of drug in the body at the steady state is independent of the rate of absorption (providing the rate is not too slow to reduce drug bioavailability), whereas the maximum and minimum drug concentrations at steady state are both functions of the rate of absorption.

\section{Summary}

Pharmacokinetic analysis indicates that patients with severely impaired renal function should receive the same loading dose of digoxin as patients with normal renal function. The calculated loading dose for both types of patients, however, is appreciably lower than the usually recommended loading doses in the literature. The analysis has indicated that the patients with severely impaired renal function should be administered maintenance doses of digoxin which are only about one half those administered to patients with normal renal function. Under these conditions, both types of patients will have equal average amounts of digoxin in the body (and in compartment 2 of the twocompartment open model) at the steady state. To achieve equal average steadystate plasma concentrations, the maintenance dose of the patients with severely impaired renal function would have to be one third of the maintenance dose administered to patients with normal renal function; the loading dose would have to be about five times the maintenance dose for the severely impaired renal patients, and about two and a half times the maintenance dose for the patients with normal renal function. Thus, using the plasma concentration criterion, the loading dose in patients with severely impaired renal function would be two thirds of the loading for patients with normal renal function, since

$$
\frac{5.0 \times 0.33}{2.5 \times 1}=\frac{1.65}{2.5} \simeq 0.67 .
$$

\section{References}

1. Jelliffe, R. W.: An improved method of digoxin therapy. Ann. Int. Med. 69:703 (1968).

2. Chung, E. K.: Digitalis Intoxication. Baltimore, The Williams and Wilkins Company, 1968, p. 18.

3. Reuning, R. H., Sams, R. A., and Notari, R. E.: Role of pharmacokinetics in drug dosage adjustment. I. Pharmacologic effect kinetics and apparent volume of distribution of digoxin. J. Clin. Pharmacol. 13:127 (1973).

4. Conn, H. F.: Current Therapy 1971. Philadelphia, W. B. Saunders Company, 1971.

5. Ogilvie, R. I., and Ruedy, J.: An educational program in digitalis therapy. J.A.M.A. 222:50 (1973).

6. Wagner, J. G., Christensen, M., Sakmar, E., Blair, D., Yates, J. P., Willis, P. W., III, Sedman, A J., and Stoll, R. G.: Equival- 


\section{WAGNER}

ence lack in digoxin plasma levels. J.A.M.A. $224: 199$ (1973).

7. Lindenbaum, J., Butler, V. P., Jr., Murphy, J. E., and Cresswell, R. M.: Correlation of digoxin-tablet dissolution rate with biological availability. Lancet I:1215 (1973).

8. Smith, T. W.: Drug therapy. Digitalis glycosides. New England J. Med. 288: 719 (1973).

9. Marcus, F. I., Pavlovich, J., Burkhalter, L., and Cuccia, C.: The metabolic fate of tritiated digoxin in the dog: a comparison of digitalis administration with and without a loading dose. J. Pharmacol. Exp. Therap. 156:548 (1967).

10. Wagner, J. G., Northam, J. I., Alway, C. D., and Carpenter, O. S.: Blood levels of drug at the equilibrium state after multiple dosing. Nature 207:1301 (1965).

11. Gibaldi, M., Nagashima, R., and Levy, G.: Relationship between drug concentration in plasma or serum and amount of drug in the body. J. Pharm. Sci. 58:193 (1969).

12. Perrier, D., and Gibaldi, M.: Relationship between plasma or serum concentration and amount of drug in the body at steady state upon multiple dosing. $J$. Pharmacokinet. Biopharm. 1:17 (1973).

13. Wagner, J. G.: Drug accumulation. J. Clin. Pharmacol. 7:84 (1967).

14. Krüger-Thiemer, E.: Formal theory of drug dosage regimens, Part I. J. Theoret. Biol. 13:212 (1966).

15. Krüger-Thiemer, E., and Pellichan, E. W.: Qualitative and quantitative definitions of drug accumulation. Pharmacologist 10: 166 (1968).

Please address all correspondence to Dr. John G. Wagner, Upjohn Center for Clinical Pharmacology, The University of Michigan, Ann Arbor, Mich. 48104. 